\title{
A satisfação e as intenções comportamentais dos sócios-torcedores de um clube pernambucano em relação aos serviços prestados no campeonato brasileiro de futebol - série $A$
}

\author{
Victor Henrique Rodrigues Silva' \\ Corlos Augusto Mulatinho de Queiroz Pedroso² \\ Yves de Holanda Batista de Miranda ${ }^{3}$ \\ Marcos Antônio Barros Filho ${ }^{4}$ \\ José Pedro Sormento 5
}

\section{RESUMO}

O objetivo deste estudo foi analisar a satisfação e as intenções comportamentais dos sócios-torcedores de um clube de Pernambuco no Campeonato Brasileiro de Futebol Série A. O estudo é caracterizado como pesquisa descritiva, com a população composta por sócios-torcedores do clube. Os dados foram coletados por meio de questionário de escala multi-itens do tipo Likert com cinco alternativas. Os dados foram analisados no software IBM SPSS Statistics 20, através da estatística descritiva, utilizando média, moda, mediana, desvio padrão, soma, medidas percentuais e gráficos. Os resultados apresentam números positivos em relação à satisfação com os serviços auxiliares, indiferença em relação ao jogo e resultados positivos nas intenções comportamentais. Conclui-se que houve resultados positivos em relação à qualidade de serviço, equilíbrio

1 Bacharel em Educação Física pela Escola Superior de Educação Física da Universidade de Pernambuco (ESEF-UPE). Recife/Pernambuco, Brasil. E-mail: victorhenrique47@hotmail.com

2 Mestre em Gestão Desportiva. Doutorado em andamento em Educação Física. Professor Assistente da Universidade de Pernambuco (UPE). Recife/Pernambuco, Brasil. E-mail: carlosaugustomulatinho@gmail.com

3 Bacharel em Educação Física pela Escola Superior de Educação Física da Universidade de Pernambuco (ESEF-UPE). Recife/Pernambuco, Brasil. E-mail: miranda95y@gmail.com

4 Bacharel em Educação Física. Mestrado em andamento em Gestão Desportiva pela Faculdade de Desporto da Universidade do Porto (FADEUP). Porto, Portugal. E-mail: marcos.barrosf@hotmail.com

5 Doutor em Ciências do Desporto. Professor Associado da Universidade do Porto (UP). Porto, Portugal. E-mail: psarmento@fade.up.pt 
quando se tratou da satisfação com os jogos e resultados bastante positivos quando se questionou sobre as intenções de consumir mais produtos e serviços do clube na visão de seus sócios-torcedores entrevistados.

Palavras-chave: Gestão esportiva. Marketing esportivo. Satisfação. Lealdade. Futebol

\title{
The satisfaction and the behavioral intention of the supportive member of a Pernambuco club in relation to the services provided
}

\begin{abstract}
The objective of this study was to analyze the satisfaction and behavioral intentions of the supporters of a Pernambuco club in the Brazilian Football Championship - Serie A. The study is characterized as descriptive research, with the population made up of club members-supporters. The data were collected through a multi-item Likert scale questionnaire with five alternatives. The data were applied in IBM SPSS Statistics 20 software, analyzing them in a descriptive way, using mean, fashion and median, standard deviation, sum, percentage measures and graphs. The results present positive numbers in relation to the satisfaction with the auxiliary services, indifference in relation to the game and positive results in the behavioral intentions. It was concluded that there were positive results regarding the quality of service, balance when it was the satisfaction with the games and very positive results when asked about the intentions to consume more products and services of the club in the vision of its partner-fans interviewed.
\end{abstract}

Keywords: Sports management. Sports marketing. Satisfaction. Loyalty. Football

\section{La satisfacción y la intención conductual del socio hincha de un club pernambucano en relación a los servicios prestados}

\section{RESUMEN}

El objetivo del estudio fue analizar la satisfacción y las intenciones comportamentales de los socios aficionados de un club de Pernambuco en el Campeonato Brasileño de Fútbol - Serie A. El estudio se caracteriza como una investigación descriptiva, con la población compuesta por socios-aficionados del club. Los datos fueron recolectados por medio de un cuestionario de escala multi-ítems del tipo Likert con cinco alternativas. Los datos fueron aplicados en el software IBM SPSS Statistics 20, analizándolos de una manera descriptiva, utilizando promedio, moda y mediana, desviación estándar, suma, medidas porcentuales y gráficos. Los resultados muestran cifras positivas en relación con la satisfacción con los servicios auxiliares, la indiferencia hacia el juego y los resultados positivos en las intenciones del comportamiento. Se concluyó que hubo resultados positivos en relación a la calidad de servicio, equilibrio cuando se trató de la satisfacción con los juegos y resultados bastante positivos cuando se cuestionó sobre las intenciones de consumir más productos y servicios del club en la visión de sus socios-aficionados entrevistados.

Palabras clave: Gestión deportiva. Marketing deportivo. Satisfacción. Lealtad. Fútbol 


\section{INTRODUÇÃO}

O marketing esportivo é definido como o processo de planejar e implementar ações que visam a produção, o preço, a comunicação e a distribuição de produtos e serviços esportivos, com foco em atingir ou superar as expectativas do torcedor em relação as metas da organização (PITTS E STOTLAR, 2002). O aumento da concorrência que os clubes de futebol começaram a ter com outras práticas de lazer e de entretenimento os levaram a investir em ações de marketing com o intuito de gerar vantagem competitiva no mercado (BISCAIA, 2016). Além da concorrência, o marketing tem importância no futebol por ser o esporte com maior aceitação nacional, atrair investidores, patrocinadores, dentre outros. Outro fator importante é que os clubes possuem um público-alvo considerado fiel em todas as competições e ações que o seu clube esteja inserido (FERREIRA, 2010). Portanto, a ligação afetiva do torcedor com o clube é um dos atributos diferenciadores deste esporte.

Para que os resultados dessa ligação sejam potencializados, no que diz respeito ao consumo, os clubes devem utilizar um princípio básico como fulcro: criar expectativas sobre seus produtos e serviços, através de ações explícitas e implícitas de marketing, transformando-os em objetos de desejo por parte de seus consumidores (KOTLER E KELLER, 2006). Quando o serviço é fornecido de maneira eficaz e causa boa impressão em quem consome, ocorre o que é denominado como qualidade do serviço (BISCAIA, CORREIA, ROSADO E MENEZES, 2014), definida como a resposta positiva que o consumidor tem quando há excelência no serviço prestado pelo clube (BISCAIA, 2016). Esse entendimento sobre a qualidade é de extrema importância para que as organizações tenham vantagens na competição com suas concorrentes (CRONIN E TAYLOR, 1994).

Para Yoshida e James (2010) os serviços são classificados como principal e auxiliar. O primeiro consiste no jogo propriamente dito, enquanto que os serviços auxiliares são definidos como a complementaridade do serviço principal (funcionários, campanhas e vantagem na associação, qualidade do estádio, pontos de alimentação adequados, etc.). Nesse contexto, a percepção da qualidade desses serviços é considerada o principal preditivo ou antecedente da satisfação e das intenções comportamentais do consumidor esportivo, no que diz respeito a retornar a consumir outros produtos e serviços (MITTAL, KUMAR E TSIROS, 1999).

Ao abordar a satisfação dos torcedores, entende-se que a sua análise é primordial para os clubes esportivos, pois a mesma atua como um antecedente das intenções comportamentais (GREENWELL, FINK E PASTORE, 2002; YOSHIDA E JAMES, 2010). Nesse sentido, a satisfação do torcedor pode ser entendida como o prazer em resposta da realização do serviço principal e/ou dos serviços auxiliares prestados durante um evento esportivo (YOSHIDA E JAMES, 2010). Ou seja, o consumidor se torna satisfeito a partir do momento em que as suas expectativas iniciais sobre determinado serviço são igualadas ou superadas.

Como visto anteriormente, tanto a qualidade de serviço, quanto a satisfação têm influências diretas nas intenções comportamentais positivas do torcedor, que se define como a intenção de voltar aos eventos, recomendar aos outros torcedores e de se manter fiel ao clube. A avaliação destas intenções comportamentais se dá por uma linha conceitual que se utiliza de duas abordagens. A primeira é a lealdade comportamental, que tem ênfase em experiências passadas de consumo. A segunda é denominada de lealdade atitudinal e consiste na preferência do consumidor em relação à determinada marca (ZINS, 2001; 
BISCAIA ET AL., 2014). Atualmente, a junção dessas duas abordagens é a mais utilizada para mensurar a lealdade do consumidor com o clube (JONES E TAYLOR, 2007).

Os estímulos de intenções de comportamentos positivas sobre os serviços em questão podem resultar na lealdade, que segundo Dwyer (2011), é definida como o forte compromisso de seus consumidores com determinada organização e que resulta nas compras regulares de produtos e serviços. Além das compras regulares citadas acima, a lealdade permite a diminuição nas despesas de captação de novos torcedores (THEYSOHN, HINZ, NOSWORTHY E KIRCHNER, 2009).

Um dos fatores que mais influenciam no comportamento do torcedor é a emoção, pois a identificação desse indivíduo com o serviço a afetam de maneira direta. Dos Santos (2012) afirma que os gestores dos clubes devem entender sobre todos os fatores que envolvem as emoções e fornecê-las de maneira positiva, com o intuito de gerar identificação e fidelidade em relação aos seus serviços.

Entende-se que o torcedor possui características muito diferentes das encontradas em outros mercados consumidores, pois há uma identificação forte com o produto principal. Porém, esta identificação não pode ser interpretada pelos clubes como um reflexo na satisfação dos torcedores (CARVALHO, MOLLETA, STINGHEN E KNAUT, 2013), nem tampouco concretizar em um comportamento de consumo frequente, sendo necessário que os clubes explorem este envolvimento como uma forma de gerar receitas (LAJE, 2009).

Portanto, o objetivo do estudo foi analisar a satisfação e as intenções comportamentais dos sócios-torcedores de um clube de Pernambuco no Campeonato Brasileiro de Futebol - Série A.

\section{MÉTODOS}

Por descrever as características de determinado mercado e estimar o comportamento de uma população específica, o presente estudo é caracterizado como pesquisa descritiva, por se tratar de uma coleta de dados advinda da extração de apenas uma amostra em um momento. O delineamento da pesquisa foi definido como transversal (MALHOTRA, 2006).

Seguindo as indicações de Malhotra (2006), a amostra do estudo foi considerada como não probabilística por conveniência. A população foi composta por 100 sócios-torcedores de um clube de Pernambuco durante o Campeonato Brasileiro de Futebol Série A. Para definir o tamanho amostral, utilizou-se como referência o estudo de Hinkin (1995), que recomenda uma relação mínima entre o número de respostas e cada item do questionário como de 1: 4. Este questionário possui 18 itens $(18 \times 4=72)$. Portanto, o tamanho mínimo da amostra foi definido como $n=72$.

O questionário utilizado foi dividido em três dimensões: "satisfação em relação aos serviços auxiliares", "satisfação em relação ao serviço principal" e "intenções comportamentais do torcedor diante do clube". A elaboração do questionário constituiu-se por adaptação de dois instrumentos existentes na literatura científica sobre marketing esportivo. Para a dimensão de serviços auxiliares, foi utilizado como referência o questionário utilizado por Carvalho et al. (2013). Já para as dimensões do produto principal 
e das intenções comportamentais, teve-se como referência o questionário do estudo de Biscaia, Correia, Yoshida, Rosado e Marôco (2013). Neste questionário, todos os itens que avaliam a satisfação foram medidos através da escala multi-itens do tipo Likert com cinco alternativas, onde cada alternativa equivale às seguintes respostas: $1=$ muito insatisfeito, $2=$ insatisfeito, $3=$ indiferente, $4=$ satisfeito, $5=$ muito satisfeito.

Foi aplicado um teste piloto com professores e alunos universitários ligados à área do esporte com a finalidade de encontrar possíveis dificuldades no preenchimento do questionário e compreensão do que está sendo passado. Após a finalização do pré-teste e aplicação das devidas correções, a versão final do questionário foi aplicada aos sócios-torcedores do clube estudado por meio da plataforma online GOOGLE Docs, no período de junho a agosto de 2016. Os inquiridos receberam todas as explicações a respeito da participação voluntária e, ao estar de acordo com o termo de consentimento livre e esclarecido, a partir das normas estabelecidas pelo Comitê de Ética e Pesquisa da Universidade de Pernambuco, no qual o estudo foi submetido e aprovado (CAAE: 51598115.1.0000.5192), permitiu-se seguir para as questões do questionário.

Os dados foram analisados no software IBM SPSS Statistics 20 para encontrar a média, moda e mediana, desvio padrão e a soma dos itens das variáveis para expor cada dimensão em gráficos.

\section{RESULTADOS E DISCUSSÕES}

Tabela 1 - Perfil sociodemográfico dos entrevistados.

\begin{tabular}{lccc}
\hline $\begin{array}{l}\text { De início, como consta na Tabela 1, buscou-se identificar o perfil } \\
\text { sociodemográfico e de consumo dos torcedores entrevistados. O perfil } \\
\text { sociodemográfico foi representado por informações sobre a idade, } \\
\text { sexo, cidade onde reside e a escolaridade. }\end{array}$ & $\mathbf{N = 1 0 0}$ & \% \\
\hline Idade Média & Feminino & 27,59 & - \\
\hline Gênero & Masculino & 14 & $14 \%$ \\
& Região Metropolitana do Recife & 86 & $86 \%$ \\
\hline \multirow{2}{*}{ Cidade onde reside } & Demais cidades de Pernambuco & 91 & $91 \%$ \\
& Demais cidades de outro Estado & 6 & $6 \%$ \\
\hline Fscolaridade & Fundamental & 3 & $3 \%$ \\
\hline & Médio & 2 & $2 \%$ \\
& Pós-Graduação Lato Senso & 33 & $33 \%$ \\
\hline
\end{tabular}


Os resultados do perfil sociodemográfico foram discutidos tendo como referência o estudo de Carvalho et al. (2013), que tratou sobre a mesma temática, em outra realidade e com análises semelhantes. Em relação ao sexo, houve semelhança nos resultados, que predominou o público masculino em ambos os estudos, sendo $86 \%$ masculino e 14\% feminino no presente estudo e 93,03\% masculino e 6,97\% feminino no estudo de Carvalho et al. (2014). Sobre a cidade onde reside, houve predominância do público residente na região metropolitana no presente estudo (91\%) e no estudo de Carvalho et al. $(97,1 \%)$. Também se constatou semelhança no item de escolaridade, pois a maioria dos entrevistados cursaram até o ensino superior, sendo $44 \%$ no presente estudo e $64,2 \%$ no estudo de Carvalho et al., (2013).

A tabela 2 apresenta informações sobre o perfil de consumo, em relação a quantidade de jogos frequentados, quantidade de produtos consumidos e se estes jogos/produtos foram comprados via internet ou via física.

Tabela 2 - Perfil de consumo dos entrevistados.

\begin{tabular}{lccc}
\hline Item & & Frequência & $\%$ \\
\hline Compra ingressos na & Sim & 84 & $84 \%$ \\
bilheteria? & Não & 16 & $16 \%$ \\
\hline Compra ingressos na internet? & Sim & 38 & $38 \%$ \\
\hline & Não & 62 & $62 \%$ \\
\hline Jogos frequentados na & 1 & 8 & $8 \%$ \\
temporada & 2 & 7 & $7 \%$ \\
& 3 a 5 & 13 & $13 \%$ \\
\hline Compra produtos do clube? & Mais de 5 & 70 & $70 \%$ \\
\hline & 0 & 2 & $2 \%$ \\
\hline Quantidade de produtos & Sim & 88 & $88 \%$ \\
comprados nesta temporada & Não & 12 & $12 \%$ \\
\hline Compra produtos do clube & 1 & 26 & $26 \%$ \\
através da internet? & 2 & 21 & $21 \%$ \\
\hline & 3 a 5 & 27 & $27 \%$ \\
através da internet & Mais de 5 & 9 & $9 \%$ \\
\hline & 0 & 17 & $17 \%$ \\
\hline & Sim & 51 & $51 \%$ \\
& Não & 49 & $49 \%$ \\
\hline & 1 & 14 & $14 \%$ \\
\hline & a 5 & $65 \%$ \\
\hline
\end{tabular}


Em relação ao perfil de consumo, os sócios frequentaram os jogos do seu clube por mais de cinco vezes na temporada $(70 \%)$ e preferem comprar os seus ingressos na bilheteria $(84 \%)$ ao invés de comprar através na internet $(38 \%)$. Predominantemente, os sócios compram os produtos do clube ( $88 \%$ ) e quando questionados se compram tais produtos pela internet, houve equilíbrio nas respostas (51\% não compram e $49 \%$ compram). A quantidade de produtos comprados na internet também é menor do que a quantidade de produtos comprados em lojas físicas, pois $65 \%$ relatam não comprar nenhum produto via internet e apenas $17 \%$ não compram produtos em lojas físicas.

Como dito nos métodos, dividiram-se as questões em três grandes dimensões. $\mathrm{Na}$ tabela 3, estão as questões definidas para descrever a dimensão denominada de "satisfação em relação aos serviços auxiliares do Clube" e suas respostas.

Tabela 3 - Questões e respostas referentes à dimensão "satisfação em relação aos serviços do Clube".

\begin{tabular}{|c|c|c|c|c|}
\hline Questões & Média & Mediana & Moda & Desvio padrão \\
\hline $\begin{array}{l}\text { Q.1: Estou satisfeito com os planos de sócio } \\
\text { oferecidos pelo clube. }\end{array}$ & 3,76 & 4 & 4 & 1,043 \\
\hline $\begin{array}{l}\text { Q.2: Estou satisfeito com o atendimento aos } \\
\text { sócios realizado pelo clube. }\end{array}$ & 3,28 & 3 & 3 & 1,138 \\
\hline $\begin{array}{l}\text { Q.3: Estou satisfeito com o atendimento aos } \\
\text { torcedores realizado pelo clube }\end{array}$ & 2,96 & 3 & 3 & 1,154 \\
\hline $\begin{array}{l}\text { Q.4: Estou satisfeito com os benefícios } \\
\text { oferecidos referentes aos planos de associação }\end{array}$ & 3,38 & 3,50 & 4 & 1,204 \\
\hline $\begin{array}{l}\text { Q.5: Estou satisfeito com os preços cobrados } \\
\text { referentes aos planos de associação. }\end{array}$ & 3,70 & 4 & 4 & 1,133 \\
\hline $\begin{array}{l}\text { Q.6: Estou satisfeito com a estrutura do } \\
\text { estádio do meu clube. }\end{array}$ & 3,06 & 3 & 3 & 1,090 \\
\hline $\begin{array}{l}\text { Q.7: Estou satisfeito com os produtos } \\
\text { oferecidos pelo meu clube. }\end{array}$ & 4,01 & 4 & 4 & 1,020 \\
\hline $\begin{array}{l}\text { Q.8: Estou satisfeito com os preços destes } \\
\text { produtos oferecidos. }\end{array}$ & 2,67 & 3 & 3 & 1,074 \\
\hline $\begin{array}{l}\text { Q.9: Estou satisfeito com os pontos de vendas } \\
\text { oferecidos pelo meu clube. }\end{array}$ & 3,76 & 4 & 5 & 1,084 \\
\hline $\begin{array}{l}\text { Q.10: Estou satisfeito com as campanhas de } \\
\text { marketing do meu clube. }\end{array}$ & 4,22 & 5 & 5 & 1,079 \\
\hline $\begin{array}{l}\text { Q.11: Estou satisfeito com os locais de } \\
\text { entretenimento oferecidos pelo meu clube em } \\
\text { dia de jogos. }\end{array}$ & 3,26 & 3 & 4 & 1,186 \\
\hline $\begin{array}{l}\text { Q.12: Estou satisfeito com a qualidade e locais } \\
\text { de vendas dos alimentos proporcionados pelo } \\
\text { meu clube em dia de jogos. }\end{array}$ & 2,71 & 3 & 3 & 1,217 \\
\hline
\end{tabular}


Encontram-se resultados positivos nos aspectos referentes aos planos de associação, ao atendimento, benefícios e o seu preço cobrado (Q1, Q2, Q4 e Q5). Resultados que vão de encontro com os estudos de Carvalho et al.; Steppacher $(2013 ; 2009)$, onde os autores afirmam que os sócios-torcedores do Paraná Clube e Sport Club Internacional, respectivamente, estão satisfeitos com os planos de sócio, com o preço cobrado destes e com o atendimento realizado.

No estudo de Santos (2011), verificou-se que o relacionamento dos funcionários do clube com o torcedor é um dos fatores primordiais na satisfação do mesmo. No presente estudo, este ponto foi tratado nos itens (Q2) e (Q3), onde são encontrados resultados positivos no (Q2) e negativos no (Q3), constatando uma divergência entre a percepção do atendimento ao sócio-torcedor e ao torcedor comum. Vale ressaltar a importância do atendimento ao torcedor comum, para que o mesmo se sinta atraído para se tornar sócio e consumir outros tipos de serviços do clube.

Santos (2011) também ressalta sobre a importância da qualidade das instalações, principalmente em torneios longos, pois o conforto e a experiência positiva auxiliam no desejo de voltar a assistir uma partida seguinte. Neste estudo, a estrutura da instalação foi avaliada no item Q6 e os resultados identificaram que os torcedores entenderam este item como "indiferente".

Carvalho et al. (2013) também utilizaram análises estatísticas semelhantes, que contribuem para uma comparação com outras realidades mais próxima do presente estudo. Os autores apontam que os produtos, os pontos de venda e o estádio do Clube também estão abaixo do aceitável na opinião de seus entrevistados. Já este estudo em questão, traz resultados positivos no nível de satisfação dos produtos e os locais de vendas oferecidos (Q7 e Q9), porém, os torcedores entendem que o preço destes produtos está alto (Q8).

O resultado mais expressivo positivamente da variável foi quando os entrevistados foram questionados se o mesmo está satisfeito com as ações de marketing do seu clube (Q10). Na realidade brasileira, estudos como o de Souza e Lubisco (2006), trazem o Sport Club Internacional, clube com grande influência no cenário nacional, como o grande exemplo de sucesso quando se trata de aquisição de sócios por meio de ações de marketing.

No mesmo estudo, os autores fazem uma crítica com a realidade da maioria dos clubes brasileiros, quando se fala que as ações de marketing dos clubes do Brasil estão voltadas, na maioria das vezes, apenas para a obtenção/renovação de contratos com parceiros, enquanto as ações voltadas ao torcedor são negligenciadas. Nos dias atuais, há melhoras nas ações voltadas ao torcedor, porém, a predominância ainda são ações voltadas às manutenções e obtenções de contratos, mesmo com a literatura já afirmando a importância de ações voltadas à satisfação e à lealdade de seus torcedores (BISCAIA et al., 2014; CARVALHO et al., 2013; YOSHIDA E JAMES, 2011; 2010; FERREIRA, 2010).

Os principais pontos negativos da dimensão em questão foram sobre o atendimento do clube aos seus torcedores (Q3), o preço dos produtos fornecidos (Q8) e a qualidade dos alimentos atrelados aos seus pontos de venda nos dias de jogos (Q12). Em relação à qualidade e pontos de venda dos alimentos (Q12), sua avaliação está em consenso com o estudo de Steppacher (2009), onde a percepção sobre a qualidade dos alimentos oferecidos nos dias de jogos do Sport Club Internacional também está abaixo do aceitável. 
julho/2018

Abaixo, segue o gráfico referente à soma dos itens respondidos da dimensão que trazem resultados sobre a satisfação referentes aos serviços oferecidos pelo Clube.

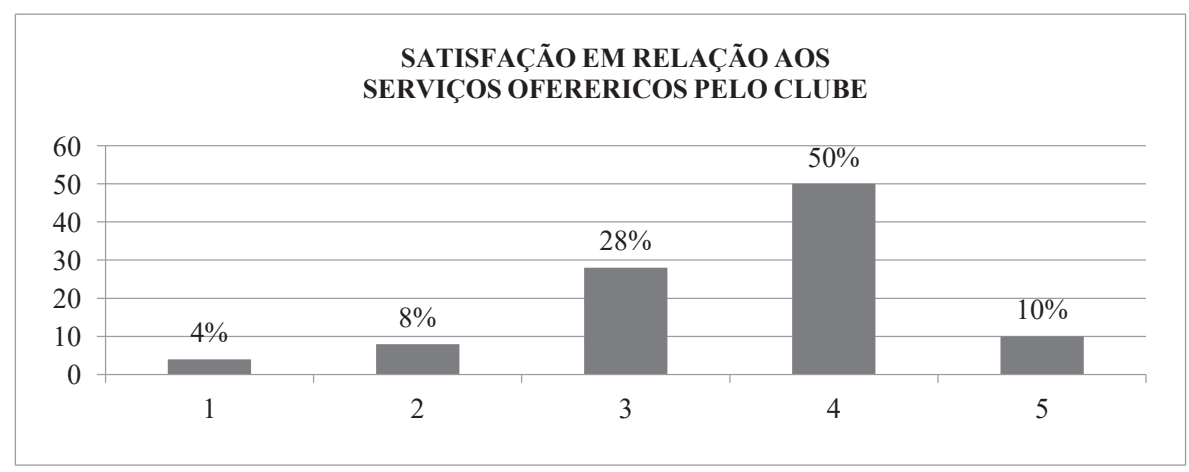

Gráfico 1 - Dimensão "satisfação aos serviços do Clube".

Após realizada a soma dos itens respondidos por cada torcedor na respectiva dimensão e apresentada em gráfico para saber o panorama geral sobre a mesma, constatou-se resultados positivos em relação aos serviços proporcionados pelo Clube em dia de jogos. A maioria dos sócios se encontram satisfeitos $(50 \%)$ e a outra parcela dos entrevistados se caracteriza como indiferentes ( $28 \%)$. Poucos foram os que se consideraram muito satisfeitos, insatisfeitos ou muito insatisfeitos (10\%, $8 \%$ e $4 \%$ respectivamente).

A próxima dimensão foi denominada de "satisfação em relação aos jogos do Clube", as questões para defini-las e suas respostas estão logo abaixo, na Tabela 4.

Tabela 4 - Questões e respostas referentes à dimensão "satisfação em relação aos jogos do Clube".

\begin{tabular}{lcccc}
\hline Questões & Média & Mediana & Moda & Desvio padrão \\
\hline $\begin{array}{l}\text { Q.13: Indique qual é o seu grau de } \\
\text { satisfação com os jogos do seu clube }\end{array}$ & 3,26 & 3 & 3 & 1,011 \\
\hline $\begin{array}{l}\text { Q.14: Indique em que medida os jogos } \\
\text { do seu clube têm correspondido às suas } \\
\text { expectativas. }\end{array}$ & 2,91 & 3 & 3 & 1,074 \\
\hline $\begin{array}{l}\text { Q.15: Indique de que forma os jogos do } \\
\text { seu clube se aproximam do jogo ideal. }\end{array}$ & 2,77 & 3 & 3 & 0,962 \\
\hline
\end{tabular}

Nesta dimensão, percebe-se um equilíbrio nos itens no que se refere aos jogos do clube em questão. Santos (2011) afirma que a imprevisibilidade que o resultado de 
um jogo de futebol tem é um dos motivos que fazem os torcedores irem ao estádio e o resultado positivo traz o prazer que o torcedor procura ao assistir uma partida de futebol. Portanto, cabe aos clubes investirem nos seus jogadores e os promoverem por meio dos seus produtos oficiais. Os clubes também devem compreender a complexidade dos seus torcedores e como eles se comportam em diferentes situações que podem acontecer em seus jogos e, a partir disso, poder potencializar as suas ações de marketing para satisfazer o seu cliente (CORREIA, BISCAIA E MENEZES, 2014).

Na visão do gestor esportivo, uma das dificuldades para um evento esportivo dar certo é a imprevisibilidade que o esporte tem. Porém, mesmo imprevisível, os clubes devem tentar minimizar esta imprevisibilidade ao máximo, pois todos os torcedores gostam e exigem que sua equipe tenha bons desempenhos e que traga resultados esportivos positivos, pois é fundamental para assegurar a satisfação dos mesmos (KUENZEL E YASSIM, 2007). Tal imprevisibilidade poderá tornar a satisfação como um processo contínuo, que talvez vá desencadear apenas no final da temporada (BISCAIA et al., 2014).

A consequência de ter torcedores satisfeitos com os jogos de sua equipe será o seu comparecimento com maior frequência aos jogos seguintes e gerar receitas por meio do lucro das bilheterias e dos serviços disponibilizados nos dias de jogos (BISCAIA et al., 2014), visto que a presença da torcida ao estádio corresponde cerca de $75 \%$ dos rendimentos dos clubes (ZHANG, SMITH E PEASE, 1996).

Abaixo, segue o gráfico 2, referente à dimensão que traz resultados sobre a satisfação do sócio torcedor com os jogos do Clube:

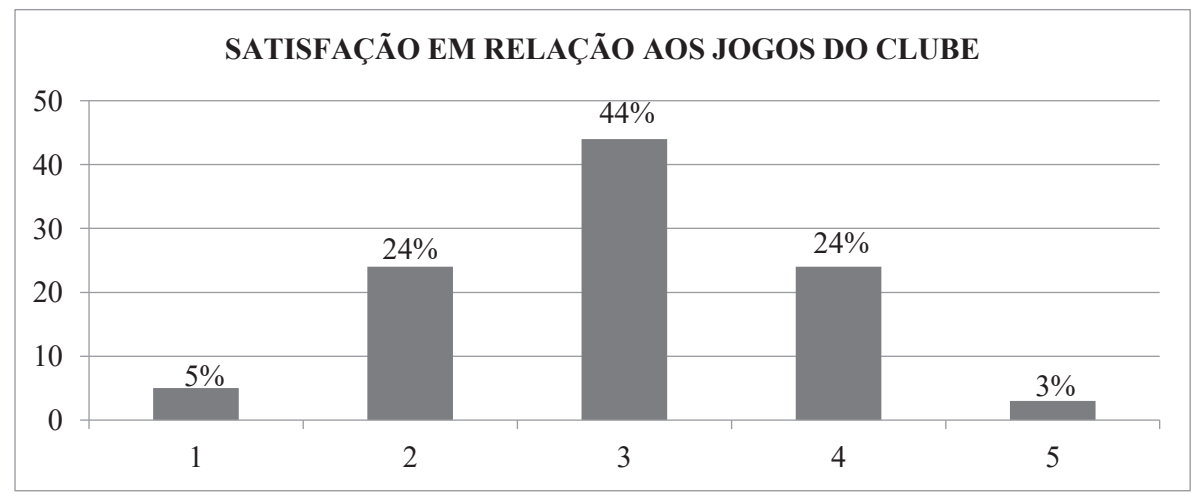

Gráfico 2 - Dimensão "satisfação em relação aos jogos do Clube".

Os resultados apresentam que a maioria dos torcedores foram classificados como indiferente (44\%) e houve equilíbrio entre os itens "insatisfeito" e "satisfeito" (24\%). Poucos foram os que se classificaram como muito insatisfeitos (5\%) ou como muito satisfeitos (3\%).

Para finalizar, definiu-se a dimensão denominada de "intenções comportamentais do torcedor diante do Clube" com as seguintes questões e respostas na tabela 5: 
julho/2018

Tabela 5 - Questões referentes à dimensão "intenções comportamentais diante do clube".

\begin{tabular}{lcccc}
\hline Questões & Média & Mediana & Moda & Desvio padrão \\
\hline $\begin{array}{l}\text { Q.16: Probabilidade de assistir a mais } \\
\text { jogos do meu clube. }\end{array}$ & 4,55 & 5 & 5 & 0,796 \\
\hline $\begin{array}{l}\text { Q.17: Intenção de comprar outros } \\
\text { produtos e serviços do meu time. }\end{array}$ & 4,31 & 5 & 5 & 1,032 \\
\hline $\begin{array}{l}\text { Q.18: Intenção de me manter sócio do } \\
\text { clube }\end{array}$ & 4,56 & 5 & 5 & 0,783 \\
\hline
\end{tabular}

Estes resultados constatam a ligação com a afirmação de Santos (2011) em relação aos produtos e serviços no clube, onde os torcedores se definiram como muito satisfeitos e que voltariam a consumi-los. O item que mediu a satisfação com os jogos do clube foi entendido como indiferente, porém, estes torcedores também definiram que é muito provável voltar aos jogos do clube mesmo com o baixo grau de satisfação encontrado na segunda dimensão do estudo, indo de acordo com a afirmação de Dwer (2011) dita anteriormente, pois as compras e consumos dos serviços de forma regular já estão demonstradas.

Ter torcedores leais é uma excelente estratégia para a diminuição dos gastos dos clubes, pois se gasta menos com a manutenção de um torcedor do que ter que captá-los de maneira recorrente (KOTLER E ARMSTRONG, 2008). Além da contenção de gastos, o lucro também aumentará em paralelo, pois os torcedores leais irão ter o hábito de consumir produtos e serviços do clube com mais frequência do que um torcedor que ainda está no processo de captação e também será mais maleável com os serviços que não atingiram sua expectativa (CHEN, SHEN E LIAO, 2009).

Santos (2011) afirma que "um investimento na qualidade funcional associado a uma melhoria na qualidade do jogo poderá ser fundamental para o desenvolvimento de novas estratégias, com vista à fidelização de novos espectadores". Cardoso (2011) também não descarta os serviços auxiliares nas intenções de comportamento, pois em seu estudo, os constrangimentos dos serviços auxiliares são diretamente ligados com as intenções de voltar à consumir produtos e participar dos eventos organizados pelo seu clube.

Para que essas ideias se concretizem, Oliver (1997) afirma que o processo de captação até a lealdade perpassa por quatro etapas: as informações que o torcedor tem de determinado clube, a satisfação por meio do consumo dos serviços e produtos do clube, desenvolvimento de uma identificação favorável com o clube e o comportamento regular de consumir produtos e serviços posteriores.

O gráfico 3 é responsável pelo panorama geral da probabilidade de o torcedor estudado continuar a consumir serviços e produtos do clube, além do evento esportivo. 


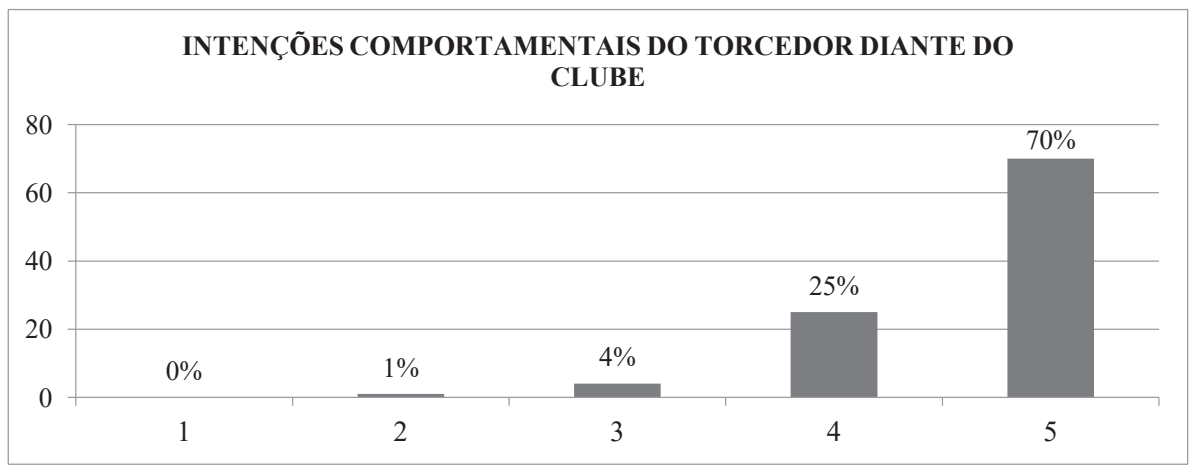

Gráfico 3 - Dimensão "intenções comportamentais do torcedor diante do clube".

Apresentam-se resultados positivos em todos os itens da variável, visto a partir do gráfico, onde $70 \%$ de seus entrevistados consideraram que é muito provável continuar consumindo serviços e produtos, assistindo aos jogos e de se manterem sócios. Os itens seguintes foram o "provável" (24\%), "indiferente" (4\%) e "improvável" (1\%).

\section{CONCLUSÕES}

Conclui-se que houveram resultados positivos em relação aos serviços auxiliares, equilíbrio quando tratou-se da satisfação com os jogos e resultados bastante positivos quando se questionou sobre as intenções de consumir mais produtos e serviços do clube na visão de seus sócios-torcedores entrevistados.

No âmbito dos serviços auxiliares, os resultados apresentados sobre as campanhas de marketing estão bem avaliadas pelos torcedores entrevistados. O marketing do clube, quando apresenta ações relacionadas com a emoção do torcedor, facilitam nas intenções futuras de consumo e isso pode explicar a alta avaliação no item referente à intenção de consumir mais produtos e serviços.

Em relação ao serviço principal, a indiferença dos torcedores não influenciou de maneira negativa à intenção de assistir à mais jogos do clube. Supõe-se que a imprevisibilidade dos resultados seja um fator motivador para a presença dos torcedores, mesmo não estando satisfeitos com o desempenho de sua equipe.

A dimensão de intenções comportamentais em relação ao clube demonstra a forte ligação entre os serviços e as emoções do torcedor. Os indivíduos entrevistados demonstraram altos níveis de lealdade, o que explana a forte identificação que o torcedor tem com o seu clube, que deve trazer ações que explorem a sua paixão.

Para tanto, sugerem-se novos estudos com objetivo de analisar a satisfação e as intenções comportamentais de torcedores em outras realidades no Brasil, bem como em eventos de outras modalidades esportivas. Além de estudos com análises estatísticas mais 
julho/2018

robustas, que apresentem, de maneira mais direta, influências de cada item nas intenções comportamentais dos torcedores.

\section{REFERÊNCIAS}

BISCAIA, R. Revisiting the Role of Football Spectators' Behavioral Intentions and its Antecedents. The Open Sports Science Journal, v. 9, p. 3-12, 2016.

BISCAIA, R. et al. Estudo da lealdade dos espectadores. Recife: UFPE, 2014.

BISCAIA, R. et al. The role of service quality and ticket pricing on satisfaction and behavioural intention within professional football. International Journal of Sports Marketing and Sponsorship, p. 42-66, 2013.

CARDOSO, J. M. A infuência dos constragimentos na lealdade dos espectadores de futebol. 2011. 150 f. Dissertação (Mestrado) - Curso de Gestão do Desporto, Faculdade de Motricidade Humana, Lisboa, 2011.

CARVALHO, W. G.; MOLLETTA, S. R.; KNAUT, F. Estudo sobre a satisfação do SócioTorcedor do Paraná Clube. Revista Intercontinental de Gestão Desportiva, p. 55-69, 2013.

CHEN, Y.; SHEN, Y.; LIAO, S. An integrated model of customer loyalty: an empirical examination in retailing practice. The Service Industries Journal, p. 267-280, 2009.

CORREIA, A.; BISCAIA, R.; MENEZES, V. Lições de marketing do desporto. Recife: UFPE, 2014.

CRONIN, J. J.; TAYLOR, S. A. SERVPERF versus SERVQUAL: reconciling performance-based perceptions minus expectations measurement of service quality. Journal of Marketing, p. 125-131, 1994.

DIAS, D. R. Gestão e Marketing. São Paulo: Saraiva, 2012.

DOS SANTOS, M. A. An Attendance Behavior Model At Sports Events: Comparison and Constrast of Two Models. Sport Science Review, v. 21, abr. 2012.

DWYER, B. Divided loyalty? An analysis of fantasy football involvement and fan loyalty to individual National Football Leagu (NFL) teams. Journal of Sport Management, p. 445-457, 2011.

FERREIRA, E. J. C. A utilização de técnicas de marketing esportivo como forma de fortalecimento da relação torcedor-clube. Um estudo sobre o CRB. Trabalho de Graduação. Maceió: Faculdade de Economia, Administração e Contabilidade, Universidade Federal de Alagoas, 2010.

GREENWELL, T.; FINK, J.; PASTORE, D. Assessing the Influence of the Physical Sports Facility on Customer Satisfaction within the Context of the Service Experience. Sport Management Review, v. 5, p. 129-148, 2002.

HINKIN, T. R. A Review of Scale Development Practices in the Study of Organizations. Journal of Management, p. 967-988, 1995.

JONES, M. V.; TAYLOR, S. F. The conceptual domain of service loyalty: How many dimensions? Journal of Services Marketing, v. 21, p. 36-51, 2007. 
KIM, D.; LAVETTER, D.; LEE, J. The influence of service quality factors on customer satisfaction and repurchase intention in the Korean professional basketball.. International Journal of Applied Sports Sciences, 2006.

KOTLER, P.; ARMSTRONG, G. Principles of Marketing. 12 $^{\mathrm{a}}$ ed. New Jersey: Prentice Hall, 2008.

KOTLER, P.; KELLER, K. L. Administração de Marketing. São Paulo: Person Prentice Hall, 2006.

KUENZEL, S.; YASSIM, M. The effect of joy on the behaviour of criket spectators: the mediating role of satisfaction. Managing Leisure, p. 43-57, 2007.

LAJE, M. J. C.. A dimensão tribal do consumo de produtos relacionados com clubes de futebol. 2009. 150 f. Dissertação (Mestrado) - Curso de Marketing, Instituto Superior de Economia e Gestão, Universidade Técnica de Lisboa, Lisboa, 2009.

LEEWEUN, L. V.; QUICK, S.; DANIEL, K. The sport spectator satisfaction model: a conceptual framework for understanding the satisfaction of spectators. Sport Management Review, 2002.

MALHOTRA, N. K. Pesquisa de markerting: uma orientação aplicada. $4^{\mathrm{a}} \mathrm{ed}$. Porto Alegre: Bookman, 2006.

MITTAL, V.; KUMAR, P.; TSIROS, M. Attribute-level performance, satisfaction, and behavioral intentions over time: A consumption-system approach. Journal of Marketing, p. 88-101, 1999.

MULLIN, B.; HARDY, S.; SUTTON, W. Sport Marketing. 3 ed. Champaign: Human Knetics, 2007.

NETO, F. M. Marketing Esportivo. Rio de Janeiro: Record, 1995.

NETO, F. M. Marketing Esportivo. 4 ed. Rio de Janeirdo: Record, 2007.

OLIVER, R. L. Satisfaction: a behavioral perspective on the consumer. New York: McGrawHill, 1997.

ROBINSON, L. Costumer expectations of sports organizations. European Sport Management Quarterly, p. 67-84, 2006.

SANTOS, L. F. S. Qualidade, satisfação e lealdade dos espectadores de futebol. 2011. 150

f. Dissertação (Mestrado) - Curso de Gestão do Desporto, Faculdade de Motricidade Humana, Universidade Técnica de Lisboa, Lisboa, 2011.

SCHWARZ, E. C.; HUNTER, J. D. Advanced theory and pratice in sport marketing. Oxford: Elsevier.

STEPPACHER, D. Estudo sobre a satisfação dos sócios do Sport Club Internacional sob a modalidade sócio contribuinte campeão do mundo. 2009. 150 f. TCC (Graduação) - Curso de Administração, Departamento de Ciências Administrativas, Universidade Federal do Rio Grande do Sul, Porto Alegre, 2011.

SUZA, A.; LUBISCO, M. Estratégia de marketing de relacionamento com seus sócios: a folosofia Alma Colorada. Think, p. 112-118, 2006.

THEODORAKIS, N. et al. Relationship between measures of service quality and satisfaction in professional sports. Managing Service Quality, 2011. 
julho/2018

THEODORAKIS, N.; ALEXANDRIS, K. Can service quality spectator's behavioral intentions in professional soccer? Managing Leisure, 2008.

THEYSOHN, S. et al. Official Supporters Clubs: The Untapped Potential of Fan Loyalty. International Journal of Sports Marketing, v. 10, p. 302-324, 2009.

TSUJI, Y.; BENNETT, G.; ZHANG, J. Consumer satisfaction with an action sports event. Sport Marketing Quarterly, 2007.

WONG, A. The role of emotional satisfaction in service encounters. Managing Service Quality, 2004.

YOSHIDA, M.; JAMES, J. Costumer satisfaction with game and service experiences: antecedents and consequences. Journal of Sport Management, 2010.

ZHANG, J.; SMITH, D.; PEASE, D. Spectator knowledge of hockey as a significant predictor of game attendance. Sport Marketing Quaterly, p. 41-48, 1996.

ZINS, A. H. Relative attitudes and commitment in customer loyalty models. Journal of Service Industry Management, v. 12, p. 269-294, 2001.

Recebido em: Junho/2017 Aprovado em: Outubro/2017 